\title{
Toxoplasmosis Infection in Pregnant Women
}

\section{ART ICLE INF O}

\section{Article Type}

Systematic Review

\section{Authors}

Saadatnia G** $P h D$

\section{How to cite this article} Saadatnia G. Toxoplasmosis Infection in Pregnant Women. Sarem Journal of Reproductive Medicine. 2017;1(3):127-131.
*Biotechnology Department, Iranian Research Organization for Science \& Technology (IROST), Tehran, Iran

\section{Correspondence}

Address: Sarem Women's Hospital, Basij Square, Phase 3, Ekbatan Town, Tehran, Iran. Postal Code: 1396956111

Phone: +98 (21) 44670888

Fax: $+98(21) 44670432$

gitasaadat@gmail.com

\section{Article History}

Received: February 9, 2016

Accepted: June 27, 2016

ePublished: August 15, 2017

\section{A B S T R A C T}

Introduction Toxoplasmosis is caused by a parasite named "Toxoplasma Gondii". One of the main consequences of toxoplasma infection in pregnant women is the transmission of infection to the fetus. Although congenital toxoplasma infection is rare, it may cause severe neurological and ophthalmic diseases that can lead to blindness. There are also chances of developing heart and brain abnormalities. The best approach is to take care of both before and during pregnancy and to teach ways to prevent toxoplasmosis. The purpose of this study was to review the general aspects of the disease, prevention methods, and methods for the diagnosis and treatment of Toxoplasma infection in pregnant women. In this study, referring to valid scientific databases such as Science Direct, PubMed and Elsevier, received special articles and issues related to the topic were extracted and analyzed.

Conclusion Toxoplasma infections in healthy individuals are asymptomatic or have mild and non-specific symptoms. But during pregnancy, it may be associated with serious consequences and complications, including brain and eye damage, growth retardation and even intrauterine death. Therefore, it seems necessary to give required information about prevention of toxoplasma infection during pregnancy to pregnant women or women who are planning to become pregnant.

Keywords Toxoplasmosis; Congenital Toxoplasmosis; Infection; Pregnancy; Diagnosis

\section{CIT A T I O N L I N KS}

[1] A review on human ... [2] Toxoplasma gondii: the model ... [3] Biology and epidemiology of Toxoplasma gondii in ... [4] Toxoplasmosis [5] Characterization of bifurcating structure of blood vessels using fractal ... [6] Environmental exposure of pregnant women to infection with Toxoplasma gondii-state of ... [7] Risk factors for Toxoplasmagondiiinfection in the... [8] Congenital toxoplasmosis in France in 2007: Firstresults from a national surveillance ... [9] Congenital toxoplasmosis--prenatal aspects of Toxoplasma gondii ... [10] Toxoplasmosis [11] Toxoplasmosis ... [12] Congenital ... [13] Transmission of toxoplasmosis (Toxoplasma gondii) by ... [14] Toxoplasmosis in pregnancy: Prevention, screening, and ... [15] Congenital ... [16] Toxoplasmosis: Diagnosis, treatment, and prevention in congenitally exposed ... [17] Vaccines against Toxoplasma gondii: challenges and ... [18] Immunogenetics of Toxoplasma gondii informs vaccine ... [19] Vaccination against Toxoplasma gondii: An increasing priority for collaborative ... [20] Prevalence of acute toxoplasmosis infection among 41,112 pregnant women and the mother-to-child transmission rate in a public hospital in ... [21] Survey of European programmes for the epidemiological surveillance of congenital ... [22] First Colombian multicentric newborn screening for congenital ... [23] Congenital ... [24] Laboratory diagnosis of Toxoplasma gondii infection and ... [25] 5 Toxoplasmosis in ... [26] False-positive results in immunoglobulin M (IgM) toxoplasma antibody tests and importance of confirmatory testing: The Platelia Toxo IgM ... [27] Toxoplasma gondii excretory secretory antigenic proteins of diagnostic ... [28] Toxoplasma gondii serology in pregnant woman: Characteristics and ... [29] A Toxoplasma gondii $10 \mathrm{kDa}$ in vitro excretory secretory antigen reactive with human IgM and IgA ... [30] Serodiagnosis of recently acquired Toxoplasma gondii infection in pregnant women using enzymelinked immunosorbent assays with a recombinant dense granule GRA6 ... [31] Recombinant proteins in the diagnosis of ... [32] Use of dense granule antigen GRA6 in an immunoglobulin G avidity test to exclude acute Toxoplasma gondii infection during ... [33] Evaluation of protective effect of recombinant dense granule antigens GRA2 and GRA6 formulated in monophosphoryl lipid A (MPL) adjuvant against Toxoplasma chronic infection ... [34] Molecular diagnosis of ... [35] Management of Toxoplasma gondii infection during pregnancy ... [36] Long-term impact of treated congenital toxoplasmosis on quality of life and visual ... [37] Why prevent, diagnose and treat congenital toxoplasmosis? 
موقعيت جغرافيايى و عادات غذايى در نقاط مختلف جهان متفاوت

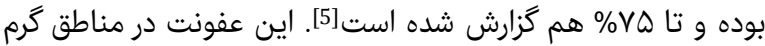

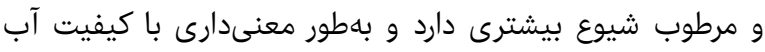

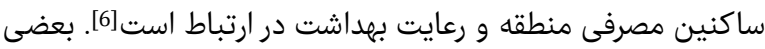

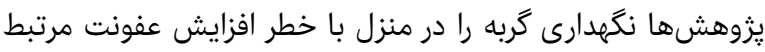

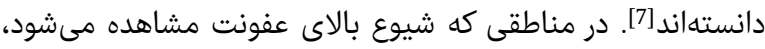

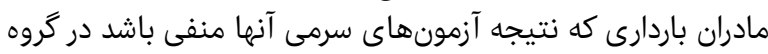

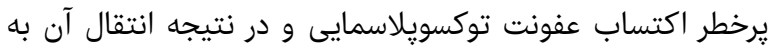

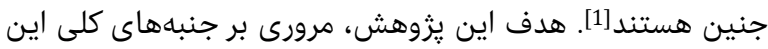

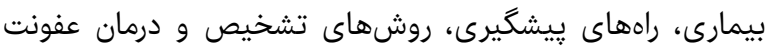
توكسويلاسمايى در زنان باردار بود.

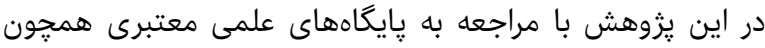

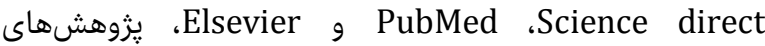
اختصاصى با كليدوازههاى عفونت توكسويلاسمايى، توكسويلاسموز

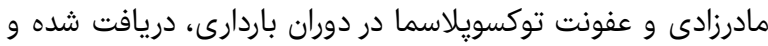
مطالب مرتبط با موضوع استخراج و مورد بررسى قرار گرفرتي.

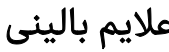

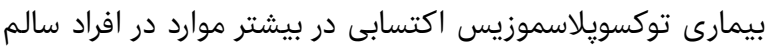

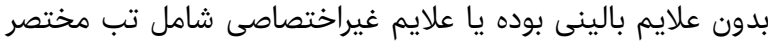

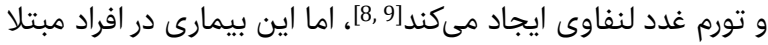

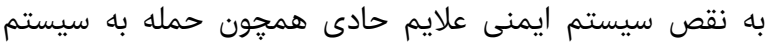

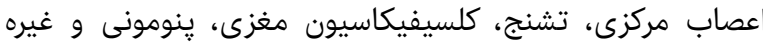

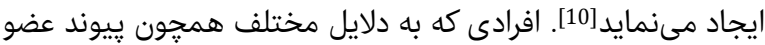

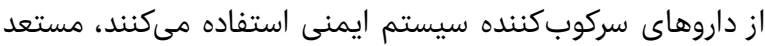

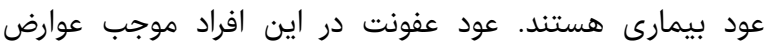

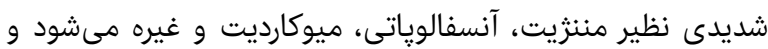

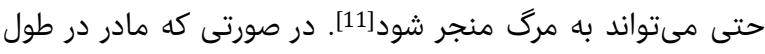

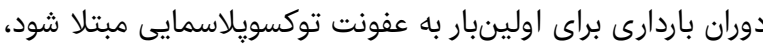

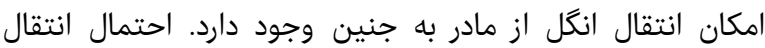

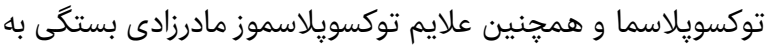

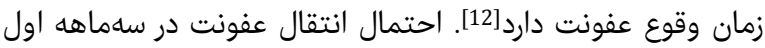

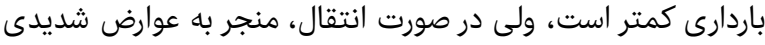

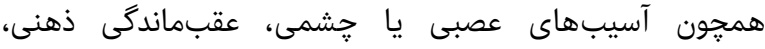

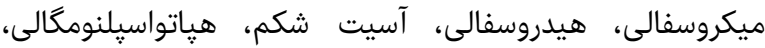

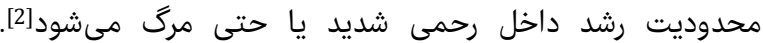

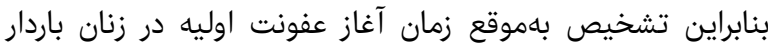
هميت خاصى دارد.

ويشخيرى

در بيشتر نقاط دنيا مصرف كوشت نيميز إيز حاوى كيستهاى انكل،

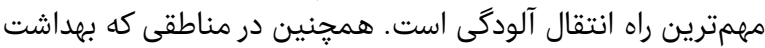

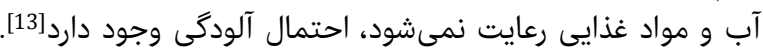

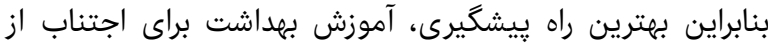

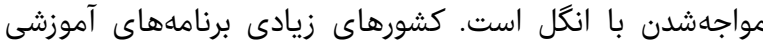

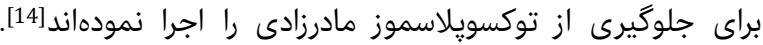

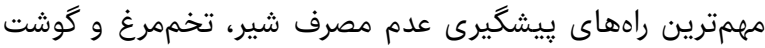

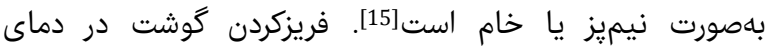

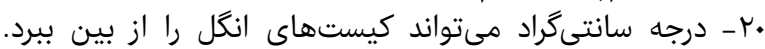

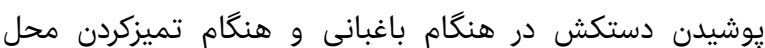

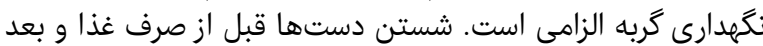

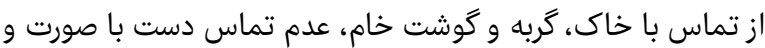

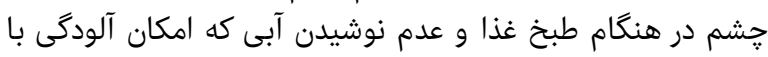

\section{عفونت توكسويلاسمايى در زنان باردار}

" PhD "

يزوهشكده زيستفناورى، سازمان يزوهشهاى علمى و صنعتى ايران، تهران، ايران

جكيده (Toxoplasma gondii)

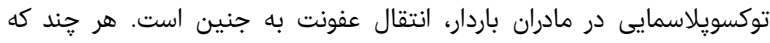

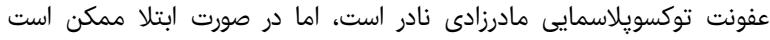

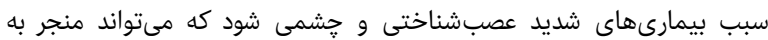

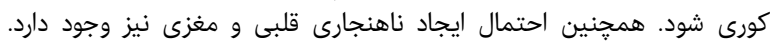

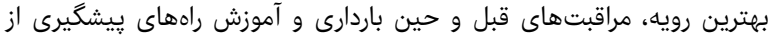

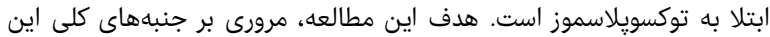

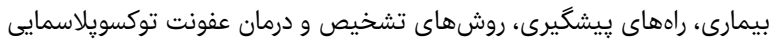

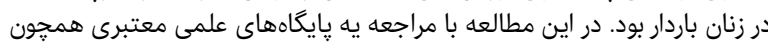

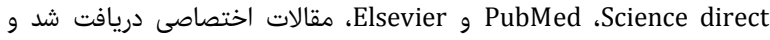
مطالب مرتبط با موضوع استخراج و مورد تحليل قرار گرفت.

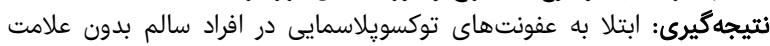

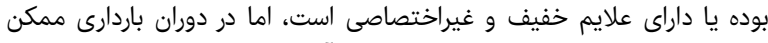

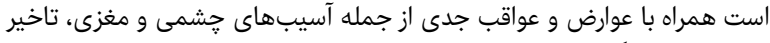

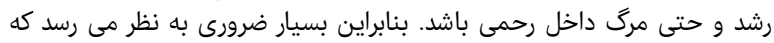

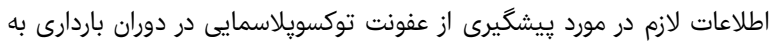

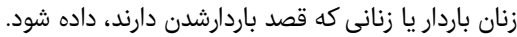

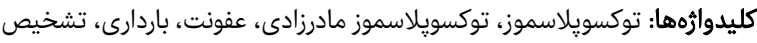

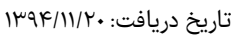

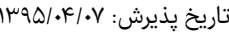
ونويسنده مسئول: gitasaadat@gmail.com

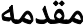

توكسويلاسموز يكى از شايعترين عفونتهاى مشترك بين انسان و و و

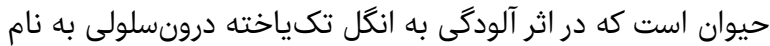

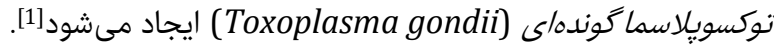

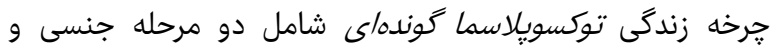

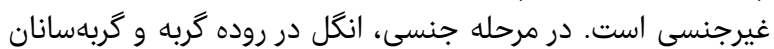

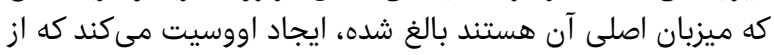

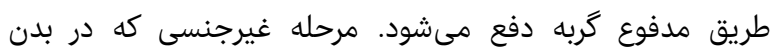

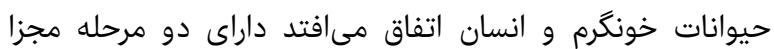

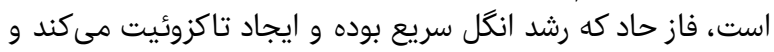

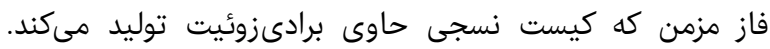

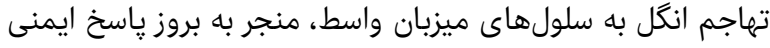

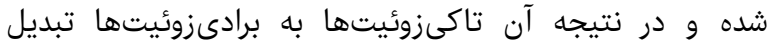

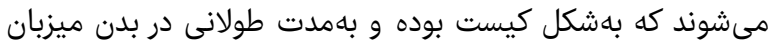

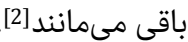

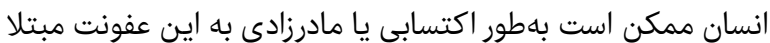

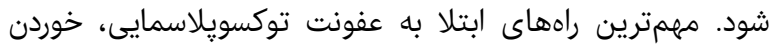

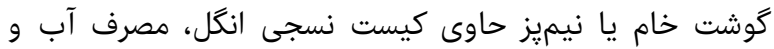

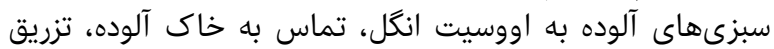

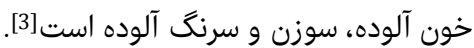

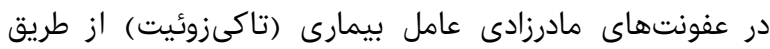

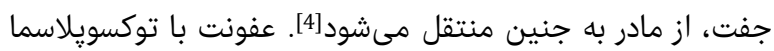
در سراسر جهان كزارش شده است، اما شيوع آلودگى بسته به بـ سن، 


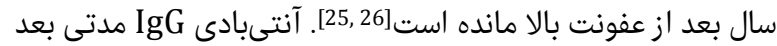

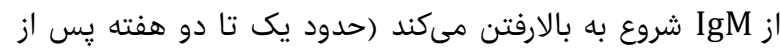

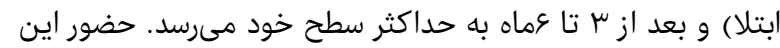

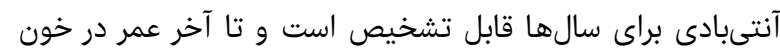

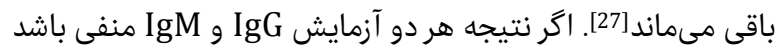

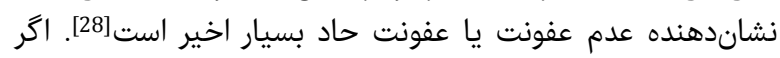

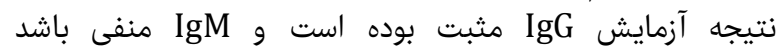

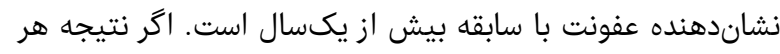

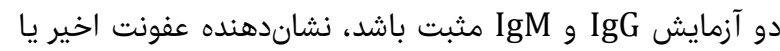

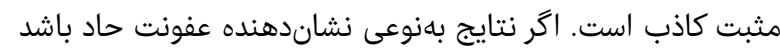

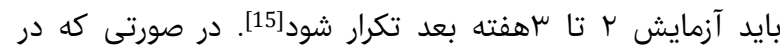

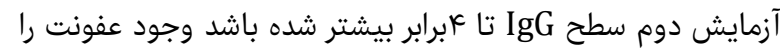

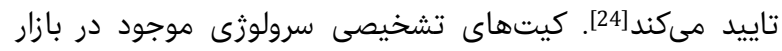

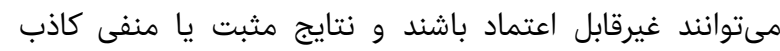

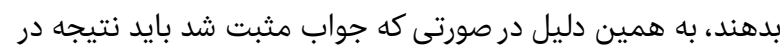

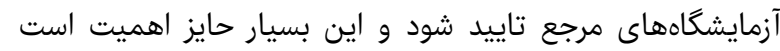

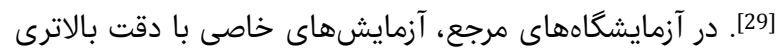

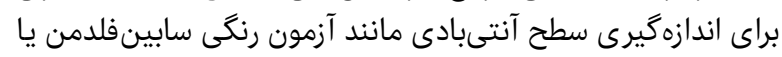

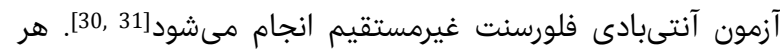

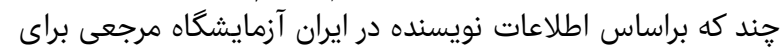

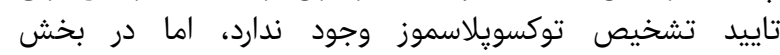

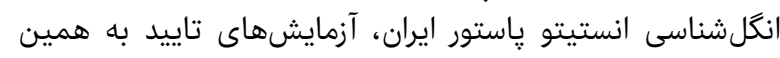

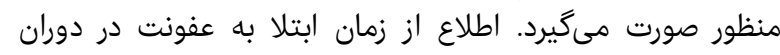

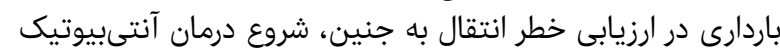

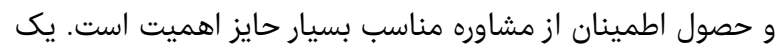

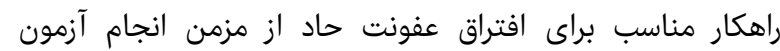

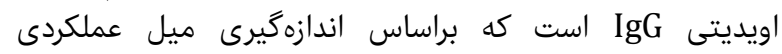

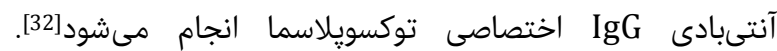

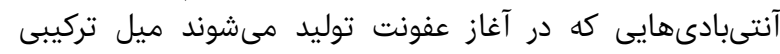

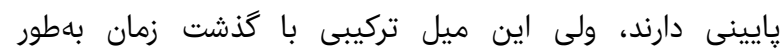

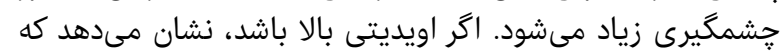

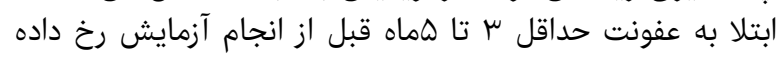

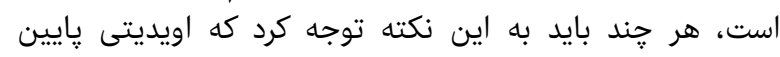

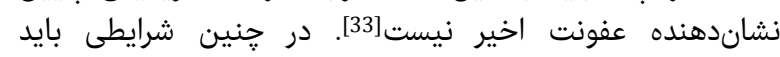

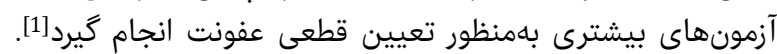

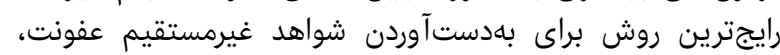

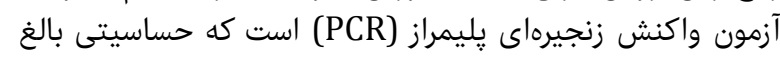

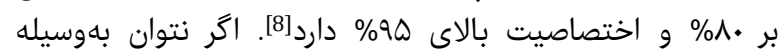

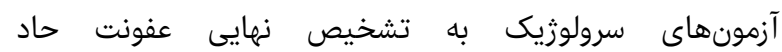

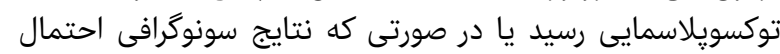

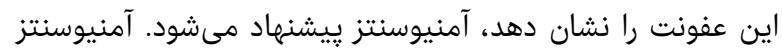

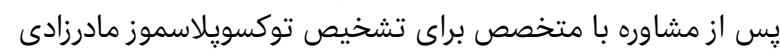

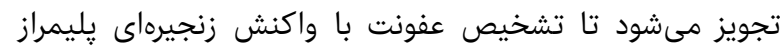

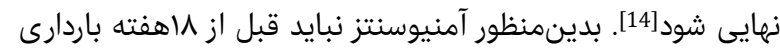

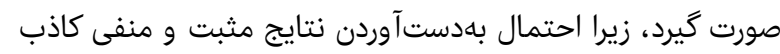

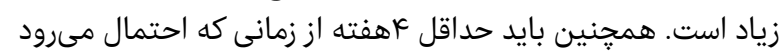

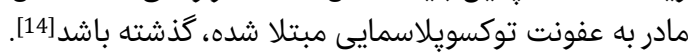

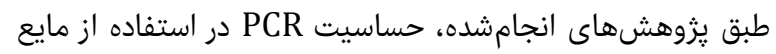

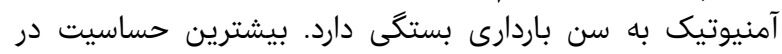

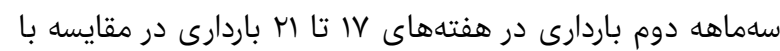

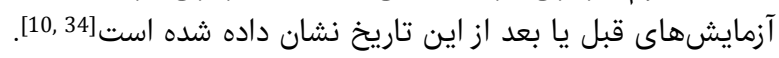

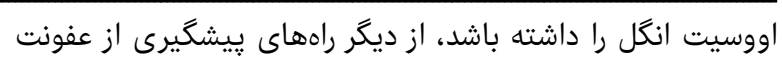

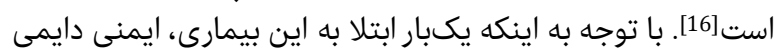

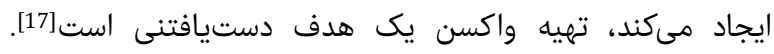

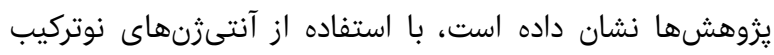

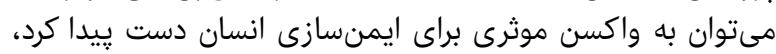
ولى اين هدف تاكنون محقق نشده است [18,

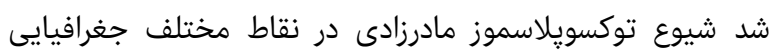

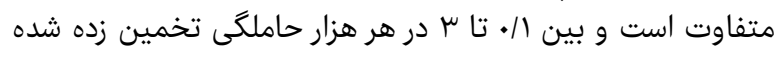

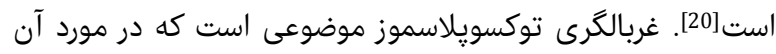

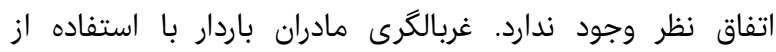

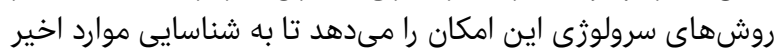

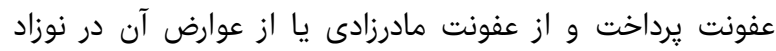

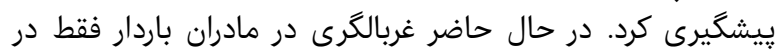

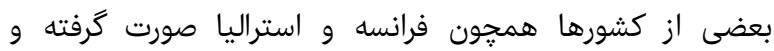

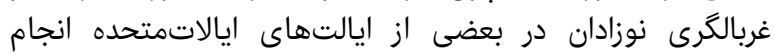

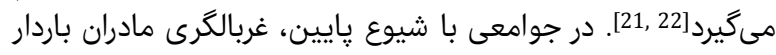

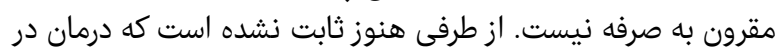

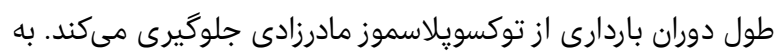

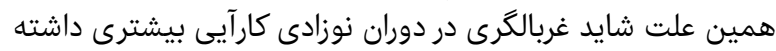

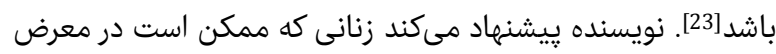

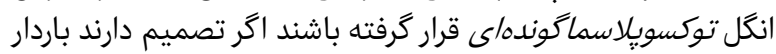

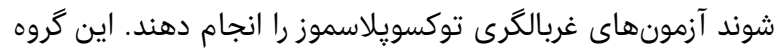

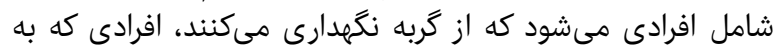

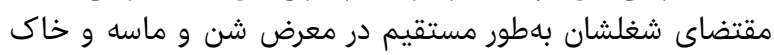

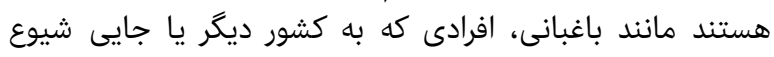

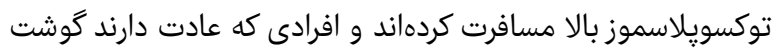
نيميز مصرف كنند.

تشخيص

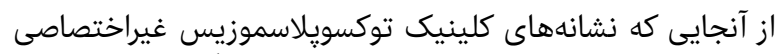

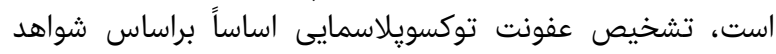
مستقيم كه نشاندهنده حضور انكل (روش ايمونويراكسيداز) يا نائ DNA

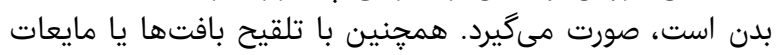

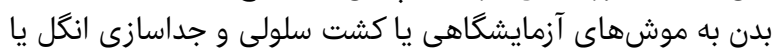

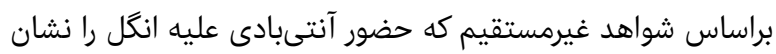

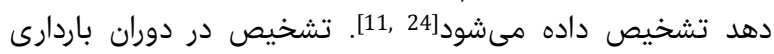

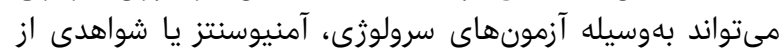

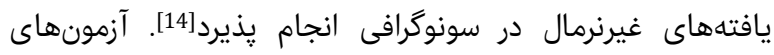

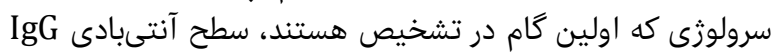

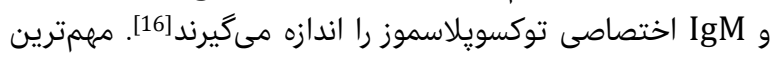

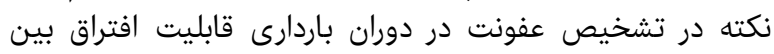

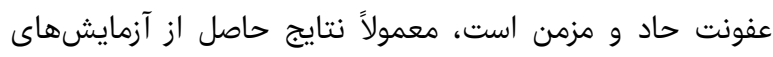

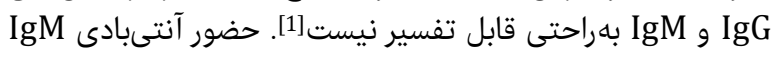

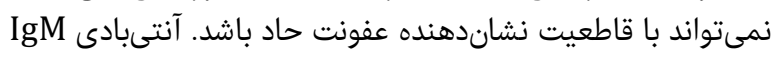

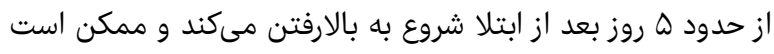

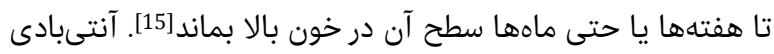

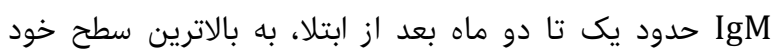

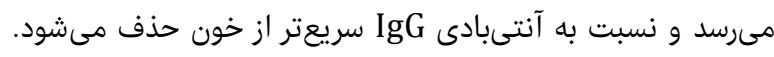

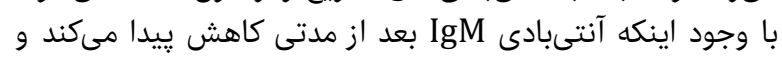

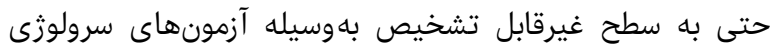
مىرسد، مواردى كزارش شده است كه سطح اين آنتىبادى تا هند 
عفونت حاد را تاييد يا رد كند و سوم، در صورتى كه در سونوكرافى مئى

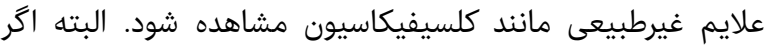

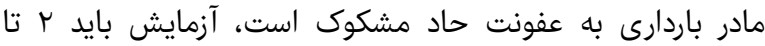

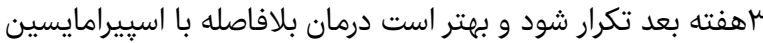

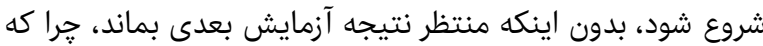

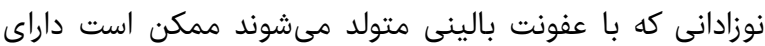

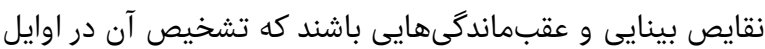

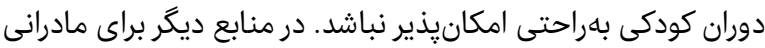

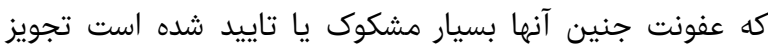

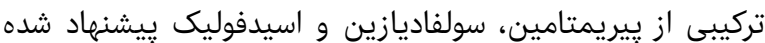

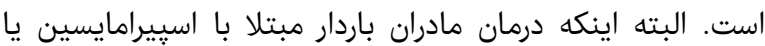

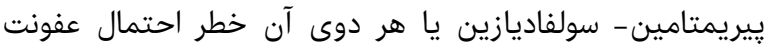

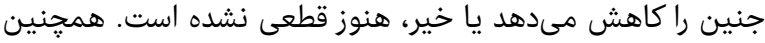

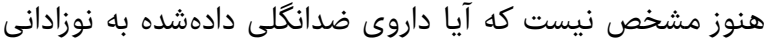

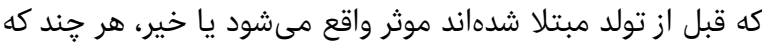

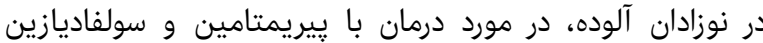

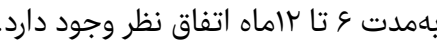

$$
\begin{aligned}
& \text { تشكر و قدردانى: موردى از سوى نويسندكان ذكر نشده است. }
\end{aligned}
$$

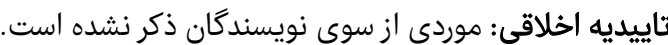

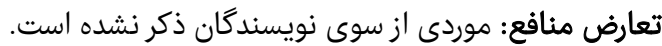

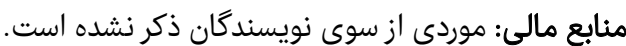

$$
\begin{aligned}
& \text { سهم نويسندكان: موردى از سوى نويسندكان كزارش نوان نشده است. }
\end{aligned}
$$

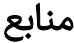

1- Saadatnia G, Golkar M. A review on human toxoplasmosis. Scand J Infect Dis. 2012;44(11):805-14.

2- Weiss LM, Kim K. Toxoplasma gondii: the model apicomplexan. Int J Parasitol. 2004;34(3):423-32.

3- Hill DE, Chirukandoth S, Dubey JP. Biology and epidemiology of Toxoplasma gondii in man and animals. Anim Health Res Rev. 2005;6(1):41-61.

4- Halonen SK, Weiss LM. Toxoplasmosis. Handb Clin Neurol. 2013;114:125-45.

5- Pappas G, Roussos N, Falagas ME. Toxoplasmosis snapshots: Global status of Toxoplasma gondii seroprevalence and implications for pregnancy and congenital toxoplasmosis. Int J Parasitol. 2009;39(12):1385-94.

6- Bojar I, Szymanska J. Environmental exposure of pregnant women to infection with Toxoplasma gondii-state of the art. Ann Agric Environ Med. 2010;17(2):209-14.

7- Jones JL, Dargelas V, Roberts J, Press C, Remington JS, Montoya JG. Risk factors for Toxoplasma gondii infection in the United States. Clin Infect Dis. 2009;49(6):878-84.

8- Villena I, Ancelle T, Delmas C, Garcia P, Brezin AP, Thulliez $\mathrm{P}$, et al. Congenital toxoplasmosis in France in 2007: First results from a national surveillance system. Euro Surveill. 2010;15(25):19600.

9- Rorman E, Zamir CS, Rilkis I, Ben-David H. Congenital toxoplasmosis--prenatal aspects of Toxoplasma gondii infection. Reprod Toxicol. 2006;21(4):458-72.

10- Montoya JG, Liesenfeld O. Toxoplasmosis. Lancet 2004;363(9425):1965-76.

11- Remington JS, R McLeod, P Thulliez, G Desmonts. Toxoplasmosis.Ch. 31. In: Remington JS, Klein JO, Wilson CB, Baker CJ. Infectious Diseases of Fetus and Newborn Infant, 6tn edition. Philadelphia: Elsevier-Saunders; 2006. p.947-1091.

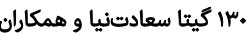
نمونه خون (از بند ناف) كه قبلاً بهاعنوان بهترين روش برون براى

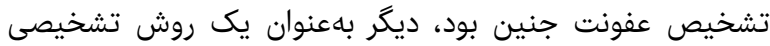

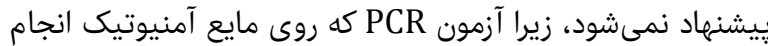

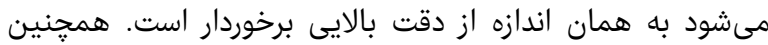
ريسك تهيه نمونه ازبند ناف بسيار بالا است [14].

درمان

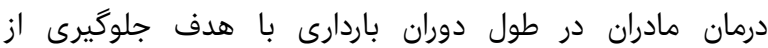

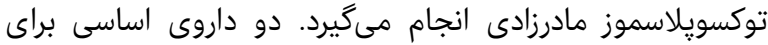

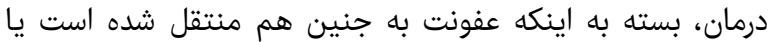

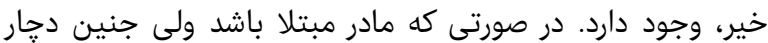

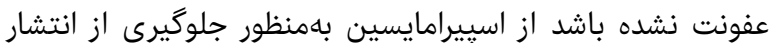

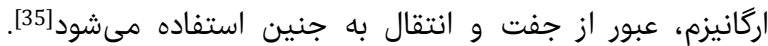

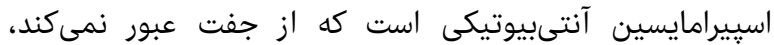

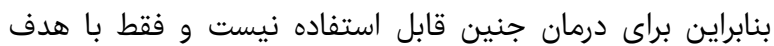

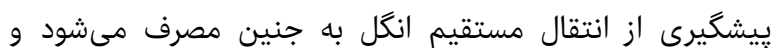

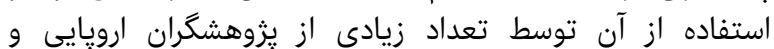

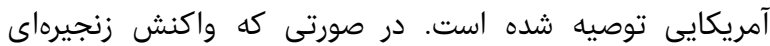

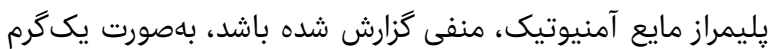

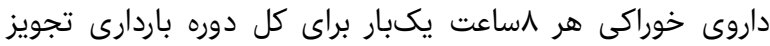

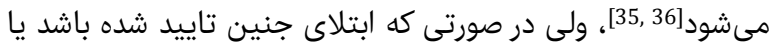

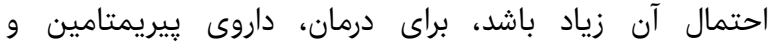

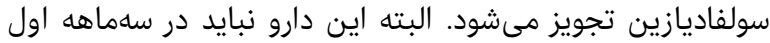

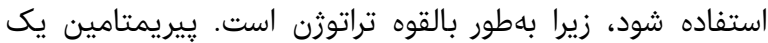

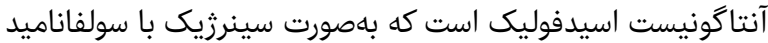

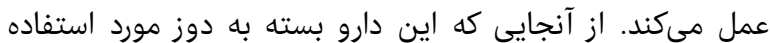

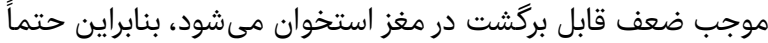

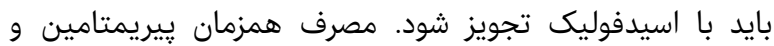

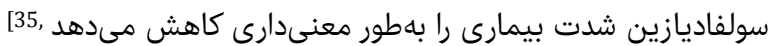

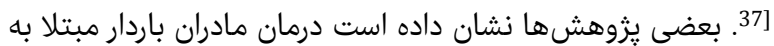

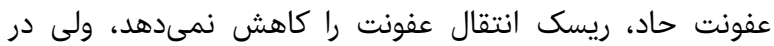

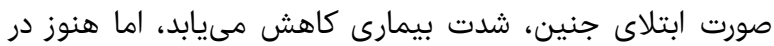

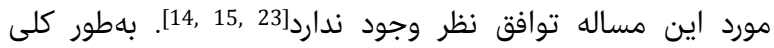

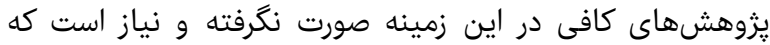

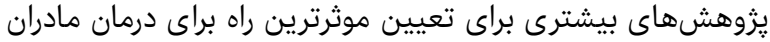

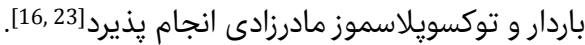

\section{نتيجه گيرى}

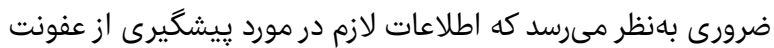

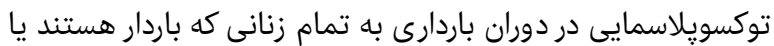

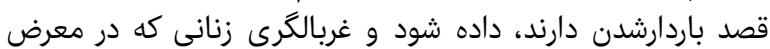

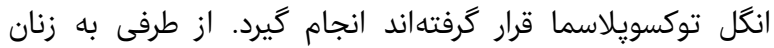

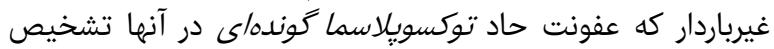

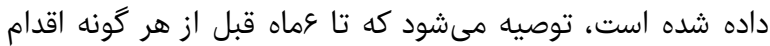

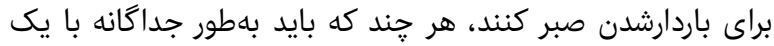

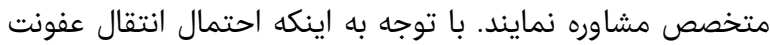

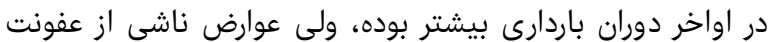

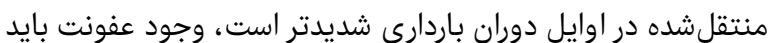

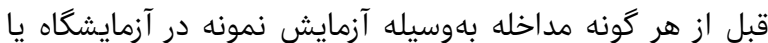

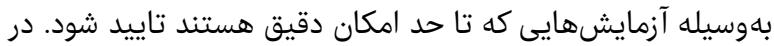

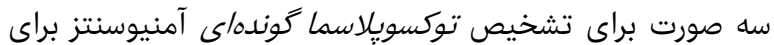

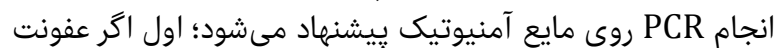

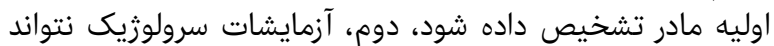


عفونت توكسويلاسمايى در زنان باردار اس|

immunoglobulin M (IgM) toxoplasma antibody tests and importance of confirmatory testing: The Platelia Toxo IgM test. J Clin Microbiol. 1997;35(1):174-8.

27 -Saadatnia G, Mohamed Z, Ghaffarifar F, Osman E, Moghadam ZK, Noordin R. Toxoplasma gondii excretory secretory antigenic proteins of diagnostic potential. APMIS. 2012;120(1):47-55.

28- Flori P, Chene G, Varlet M, Sung RT. Toxoplasma gondii serology in pregnant woman: Characteristics and pitfalls. Ann Biol Clin (Paris). 2009;67(2):125-33. [French]

29- Saadatnia G, Ghaffarifar F, Khalilpour A, Amerizadeh A, Rahmah N. A Toxoplasma gondii $10 \mathrm{kDa}$ in vitro excretory secretory antigen reactive with human IgM and IgA antibodies. Trop Biomed. 2011;28(3):606-14.

30- Golkar M, Azadmanesh K, Khalili G, KhoshkholghSima B, Babaie J, Mercier C, et al. Serodiagnosis of recently acquired Toxoplasma gondii infection in pregnant women using enzyme-linked immunosorbent assays with a recombinant dense granule GRA6 protein. Diagn Microbiol Infect Dis. 2008;61(1):31-9.

31- Kotresha D, Noordin R. Recombinant proteins in the diagnosis of toxoplasmosis. APMIS. 2010;118(8):529-42.

32- Elyasi $H$, Babaie J, Fricker-Hidalgo $H$, BrenierPinchart MP, Zare M, Sadeghiani G, et al. Use of dense granule antigen GRA6 in an immunoglobulin G avidity test to exclude acute Toxoplasma gondii infection during pregnancy. Clin Vaccine Immunol. 2010;17(9):1349-55.

33- Golkar M, Shokrgozar MA, Rafati S, Musset K, Assmar $M$, Sadaie R, et al. Evaluation of protective effect of recombinant dense granule antigens GRA2 and GRA6 formulated in monophosphoryl lipid A (MPL) adjuvant against Toxoplasma chronic infection in mice. Vaccine. 2007;25(21):4301-11.

34- Bastien P. Molecular diagnosis of toxoplasmosis. Trans R Soc Trop Med Hyg. 2002;96(Suppl 1):S205-15.

35- Montoya JG, Remington JS. Management of Toxoplasma gondii infection during pregnancy. Clin Infect Dis. 2008;47(4):554-66.

36- Peyron F, Garweg JG, Wallon M, Descloux E, Rolland

M, Barth J. Long-term impact of treated congenital toxoplasmosis on quality of life and visual performance. Pediatr Infect Dis J. 2011;30(7):597-600.

37- McLeod R, Kieffer F, Sautter M, Hosten T, Pelloux H. Why prevent, diagnose and treat congenital toxoplasmosis? Mem Inst Oswaldo Cruz. 2009;104(2):320-44.
12- Kieffer F, Wallon M. Congenital toxoplasmosis. Handb Clin Neurol. 2013;112:1099-101.

13- Pereira KS, Franco RM, Leal DA. Transmission of toxoplasmosis (Toxoplasma gondii) by foods. Adv Food Nutr Res. 2010;60:1-19.

14- Paquet C, Yudin MH, Allen VM, Bouchard C, Boucher $\mathrm{M}$, Caddy $\mathrm{S}$, et al. Toxoplasmosis in pregnancy: Prevention, screening, and treatment. J Obstet Gynaecol Can. 2013;35(1):78-81.

15- Jones J, Lopez A, Wilson M. Congenital toxoplasmosis. Am Fam Physician. 2003;67(10):2131-8.

16- Kaye A. Toxoplasmosis: Diagnosis, treatment, and prevention in congenitally exposed infants. J Pediatr Health Care. 2011;25(6):355-64.

17- Jongert E, Roberts CW, Gargano N, Förster-Waldl E, Petersen E. Vaccines against Toxoplasma gondii: challenges and opportunities. Mem Inst Oswaldo Cruz. 2009;104(2):252-66.

18- Henriquez FL, Woods S, Cong H, McLeod R, Roberts CW. Immunogenetics of Toxoplasma gondii informs vaccine design. Trends Parasitol. 2010;26(11):550-5.

19- Innes EA. Vaccination against Toxoplasma gondii: An increasing priority for collaborative research?. Expert Rev Vaccines. 2010;9(10):1117-9.

20- Varella IS, Canti IC, Santos BR, Coppini AZ, Argondizzo LC, Tonin C, et al. Prevalence of acute toxoplasmosis infection among 41,112 pregnant women and the mother-to-child transmission rate in a public hospital in South Brazil. Memórias do Instituto Oswaldo Cruz. 2009;104(2):383-8.

21 -Bénard A, Petersen E, Salamon R, Chêne G, Gilbert R, Salmi LR, et al. Survey of European programmes for the epidemiological surveillance of congenital toxoplasmosis. Euro Surveill. 2008;13(15). pii:18834.

22- Gómez-Marin JE, de-la-Torre A, Angel-Muller E, Rubio J, Arenas J, Osorio E, et al. First Colombian multicentric newborn screening for congenital toxoplasmosis. PLoS Negl Trop Dis. 2011;5(5):e1195.

23- Kravetz J. Congenital toxoplasmosis. BMJ Clin Evid. 2013;pii:0906.

24- Montoya JG. Laboratory diagnosis of Toxoplasma gondii infection and toxoplasmosis. J Infect Dis. 2002;185(Suppl 1):S73-S82.

25- Stray-Pedersen B. 5 Toxoplasmosis in pregnancy. Baillière Clin Obstet Gynaecol. 1993;7(1):107-37.

26- Liesenfeld O, Press C, Montoya JG, Gill R, IsaacRenton JL, Hedman K, et al. False-positive results in 